\title{
REVISIÓN DE LA PERTINENCIA DE LA EDUCACIÓN UNIVERSITARIA EN TERAPIA FÍSICA Y DEPORTIVA EN MÉXICO
}

\section{REVIEW OF THE RELEVANCE OF UNIVERSITY EDUCATION IN PHYSICAL THERAPY AND SPORTS IN MEXICO}

\author{
María Cristina Enríquez Reyna ${ }^{1}$ y Daniel Carranza Bautista ${ }^{1}$ \\ maria.enriquezryn@uanl.edu.mx; daniel.carranzabt@uanl.edu.mx \\ ${ }^{1}$ Universidad Autónoma de Nuevo León, Nuevo León, México \\ Envío original: 2021-01-25 Reenviado: 2021-05-20 Aceptado: 2021-07-07 \\ Publicado: 2021-08-10
}

Doi: https://doi.org/10.15517/pensarmov.v19i2.45525

\begin{abstract}
RESUMEN
En México existe una gran cantidad de personas que sufre de discapacidad y lesiones que no son tratadas adecuadamente ni se les da el seguimiento a falta de difusión de programas, proyectos y alternativas de atención que contribuyan a la continuidad del tratamiento y proceso de recuperación a través de la rehabilitación física. La formación universitaria específica en el área de la terapia física y deportiva está encaminada para mejorar los procesos de recuperación física funcional a través del apoyo hacia la adherencia a la práctica deportiva recreativa o competitiva. Se presenta una revisión narrativa sobre la pertinencia de la educación universitaria en terapia física y deportiva en México con énfasis en el campo laboral y el enfoque socioeconómico. Se analizan de forma crítica reportes sobre tres constructos: pertinencia social, oferta educativa y pertinencia socioeconómica. Se identifica una oferta educativa insuficiente, con un campo laboral muy amplio que incluye desde la afiliación a instituciones de salud hasta el emprendimiento de labores independiente lo que incide en bajas tasas de desempleo reportadas en los egresados. El salario mensual de los profesionistas de la fisioterapia resulta ser superior al promedio nacional. Dadas las condiciones nacionales al respecto de la formación profesional, la educación universitaria en terapia física y deportiva representa un área de oportunidad para
\end{abstract}


las instituciones educativas del país y una opción redituable para los interesados en incursionar en este tipo de ámbitos.

Palabras clave: fisioterapia deportiva, rehabilitación física, educación universitaria, México.

\begin{abstract}
In Mexico, there is many people who suffer from disabilities and injuries which are not adequately treated or followed up due to the lack of dissemination of programs, projects and care alternatives that contribute to the continuity of treatment and recovery process through of physical rehabilitation. Specific university training in physical and sports therapy is aimed at improving functional physical recovery processes through support towards adherence to recreational or competitive sports practice. A narrative review is presented on the relevance of university education in physical and sports therapy in Mexico with an emphasis on the labor field and the socioeconomic approach. Reports on three constructs are critically analyzed: social relevance, educational offer, and socioeconomic relevance. An insufficient educational offer is identified, with a very wide labor field that includes from affiliation to health institutions to the undertaking of independent work, which affects the low unemployment rates reported in graduates. The monthly salary of physiotherapy professionals turns out to be higher than the national average. Given the national conditions regarding professional training, university education in physical and sports therapy represents an area of opportunity for educational institutions in the country and a profitable option for those interested in venturing into this type of field.
\end{abstract}

Key words: sports physiotherapy, physical rehabilitation, university education, Mexico.

\title{
INTRODUCCIÓN
}

Existen diferentes organizaciones que dentro de su agenda trabajan para mejorar las condiciones de calidad de vida y el bienestar social de la población que enfrenta algún tipo de discapacidad. La fisioterapia o terapia física para la rehabilitación física ante la discapacidad constituye un medio para alcanzar parte de estos propósitos y, en consecuencia, se favorece la inclusión social de quienes enfrentan este tipo de situaciones (Landinez-Parra, Contreras-Valencia y Castro-Villlami, 2012). La Organización Mundial de la Salud (2020, párr. 2) establece que "en el mundo hay aproximadamente mil millones de 
personas con discapacidad, de las que la mayoría carece de acceso a una atención médica y a servicios de rehabilitación apropiados, especialmente en los países de ingresos bajos y medianos". Lo cual podría ser un indicador pertinente para promover un modelo de salud integral que involucre el área de la rehabilitación, la fisioterapia y la terapia física las cuales atienden a las directrices de un organismo rector a nivel mundial, además de generar una capacidad para mejorar la calidad de vida funcional de la población. En el 2011 se señaló que la discapacidad será un motivo de preocupación aún mayor, pues su prevalencia está aumentando (OMS, 2011); ante el envejecimiento poblacional y la incidencia de enfermedades no transmisibles, se estima que el riesgo de discapacidad es superior ya que todos los habitantes del mundo en algún momento de la vida podrían enfrentar algún tipo de discapacidad. Dada la prevalencia de discapacidad en México, se justifica la pertinencia de contar con personal especializado en el tratamiento de las discapacidades, el mantenimiento y mejoramiento de las capacidades funcionales en este sector y otros sectores específicos de la población.

Pineda (2018) explica que existe una problemática en México respecto al abandono y falta de atención sobre la terapia física y rehabilitación. En su reporte, el investigador argumenta que existe una gran cantidad de personas que sufren de discapacidad y lesiones que no son tratadas adecuadamente ni se les da el seguimiento a falta de difusión de programas, proyectos y alternativas de atención que contribuyan a la continuidad del tratamiento y proceso de recuperación a través de la rehabilitación física por lo que, son susceptibles a abandonar el tratamiento, lo que implica una problemática que requiere ser atendida en el ámbito de la terapia física, la fisioterapia y la rehabilitación.

En términos generales, México destaca por ser un país altamente poblado en Centroamérica y ocupa el segundo lugar por número de habitantes en Latinoamérica; cuenta con al menos 7.1 millones de habitantes que padecen alguna discapacidad (Instituto Nacional de Estadística y Geografía [INEGI], 2015). En contraste, la oferta educativa para formar profesionistas en el Nivel Superior para el área de la terapia física y rehabilitación está disponible en 104 universidades distribuida en 28 entidades federativas, donde el 32.5 $\%$ de las instituciones educativas se concentra en tan solo cuatro estados. Por otro lado, a pesar de la existencia de hospitales que atienden la terapia física y rehabilitación existe una brecha de conocimiento del médico con las actividades que desarrolla un terapeuta y lo que implica el trabajo multidisciplinario en el ámbito de la rehabilitación de los pacientes (Estrada-Gómez, Camacho, Sánchez y Ochoa, 2019); además, Estrada-Gómez et al. $(\underline{2019}$, p. 45) señalan que: "La regulación del ejercicio del fisioterapeuta en nuestro país es 
necesaria, ya que aún existen escuelas sin aval universitario que no garantizan una capacitación integral del fisioterapeuta en actividades de investigación, docencia y gestión".

En un área muy específica, la fisioterapia tiene una estrecha relación con la actividad física y el deporte. Desde su estructura organizativa con frecuencia las organizaciones deportivas optan por ofrecer diversos servicios de apoyo para brindar atención integral a los atletas, de ahí que se opta por contar con un departamento dirigido a la fisioterapia y rehabilitación de sus deportistas, esto debido a la necesidad de prevenir y atender lesiones que son generadas en los entrenamientos y competencias (Moreau y Nabhan, 2012). Asimismo, estos departamentos pueden realizar estudios del patrón del movimiento, además de la intervención e integración del deportista a su rendimiento de competencias después de una lesión, de ahí que la fisioterapia ayuda a evaluar las capacidades funcionales de un deportista (Pons, Gómez, Bueso y Lourido, 2006). Ante las implicaciones locomotoras y biomecánicas de la marcha y la carrera durante la práctica deportiva, esta actividad es estudiada por múltiples ramas de las ciencias del deporte debido al impacto articular y muscular que trae consigo el riesgo de lesiones y alteraciones en el rendimiento individual con efectos en el desempeño grupal al respecto de los deportes en equipo (Massimo-Biancardi, Luisa-Bona y Lagos-Hausheer, 2020). La fisioterapia en el ámbito deportivo ha venido a fortalecer los procesos de recuperación funcional de los atletas que han sufrido una lesión y se ha demostrado su efectividad posterior a una intervención quirúrgica (Grindem et al., 2015).

Cortés-González y Ravelo-Izquierdo (2017, p. 3) establecen que "para una rehabilitación adecuada, se necesita de un equipo multidisciplinario de cirugía, médicos y fisioterapeutas que desarrollen un papel importante en el manejo de las condiciones físicas y cognitivas para el regreso al terreno deportivo"; además, señalan que es necesario crear programas que incluyan fisioterapeutas especializados en el ámbito deportivo que optimicen el período de recuperación de las capacidades físicas y con ello el estado funcional del paciente, lo que requiere de la participación de un preparador físico o especialista en ciencias del ejercicio tal como lo propone el modelo del bienestar multidisciplinario. Atendiendo a lo anteriormente mencionado, la finalidad de este artículo es analizar la pertinencia y factibilidad de la educación universitaria en fisioterapia física y deportiva en México con relación al campo laboral y su enfoque socioeconómico.

\section{METODOLOGÍA}

Revisión de literatura narrativa que incluye la descripción cuantitativa de variables además de análisis de tres constructos de interés: pertinencia social, oferta laboral y 
pertinencia socioeconómica de la educación universitaria en terapia física y deportiva en México. En este trabajo se revisan y evalúan de forma crítica publicaciones sobre aspectos relativos a la pertinencia de la educación universitaria en el país además de investigar con profundidad la información disponible con el fin de explicar un fenómeno complejo (Thomas y Nelson, 2007). En este sentido se consultó información reciente de una base de datos estadísticos, cinco informes técnicos y 22 artículos de carácter científico publicados en los últimos 10 años. La búsqueda se realizó durante cuatro meses de septiembre a diciembre del 2020.

\section{Pertinencia social}

Se parte de la definición del constructo de la discapacidad y la necesidad de rehabilitación con relación a la relevancia social reportada en el país. Posteriormente se presenta descripción cuantitativa de la oferta educativa de la terapia física y/o deportiva.

\section{Oferta laboral}

Se consideró información de los planes de estudio publicados en línea a partir de la oferta educativa universitaria en el país. Se agruparon las opciones con mínima representatividad. Además, se describe el perfil profesional de los profesionales de la fisioterapia considerando el enfoque internacional y nacional.

\section{Pertinencia socioeconómica}

Se presenta a partir de la descripción de la demanda educativa, condición laboral, remuneración económica, oferta educativa y el retorno de la inversión.

\section{RESULTADOS}

\section{Pertinencia social}

En México existe una población aproximada de 127,540,408 habitantes en el 2020 dato estimado en base a la tendencia de los datos estadísticos proporcionados por el INEGI, dicha población presenta propensión a sufrir cualquier tipo de lesión y requerir en algún momento servicios de rehabilitación o terapia física para la recuperación de alguna patología que pudiesen sufrir a lo largo de su vida. Para la OMS (2011) "La discapacidad es parte de la condición humana. Casi todas las personas tendrán una discapacidad temporal o permanente en algún momento de sus vidas, y los que sobrevivan y lleguen a la vejez experimentarán cada vez más dificultades de funcionamiento" (p. 3). Por su parte, Padilla-Muñoz (2010) establece que "La discapacidad es una situación heterogénea que 
envuelve la interacción de una persona en sus dimensiones física o psíquica y los componentes de la sociedad en la que se desarrolla y vive" (p. 384).

Según datos del INEGI sobre la discapacidad en México (2015), la falta de prestación de servicios hace que "las personas con discapacidad sean especialmente vulnerables a las deficiencias en servicios tales como la atención de la salud, la rehabilitación, o el apoyo y la asistencia" (p. 72). El número de mujeres con algún tipo de discapacidad supera al de su contraparte masculina (3.8 millones frente a 3.3 millones de los varones); de igual forma, la discapacidad permanente o transitoria al interior de cada sexo tiene mayor presencia entre la población femenina; $6.2 \%$ de las mujeres del país viven con esta condición y en el caso de los hombres, representan 5.7\%. De acuerdo con el INEGI (2013), una persona con discapacidad es aquella que tiene alguna limitación física o mental para realizar actividades en su casa, en la escuela o trabajo, como caminar, vestirse, bañarse, leer, escribir, escuchar, etcétera. De ahí que la Encuesta Nacional de Ingresos y Gastos de los Hogares (ENIGH) (2013), señala que las personas con problemas para caminar son el tipo de discapacidad de mayor presencia (57.5\%), con dificultades para ver (32.5\%), oír (16.5\%), hablar o comunicarse (8.6\%), mental (8.1\%), atender el cuidado personal $(7.9 \%)$ y poner atención (6.5\%). Al respecto del costo económico, en un reporte sobre la discapacidad causada exclusivamente por lesiones de tránsito, se estimó un costo total de $\$ 4.941,77$ dólares por persona, resultando en un total de \$1.119.761.632,53 promedio en el 2012; esto representa un alto costo para el sistema de salud y la sociedad mexicana (Sánchez-Vallejo, Pérez-Núñez y Heredia-Pi, 2015).

Como parte de una oportunidad de abordar la problemática de la discapacidad, el Programa Nacional para el Desarrollo y la Inclusión de las Personas con Discapacidad 2014-2018 emite en su estrategia "2.1 Disminuir la discapacidad por enfermedades y lesiones, mediante detección, diagnóstico temprano, intervención oportuna y rehabilitación, en servicios de salud" (DOF, 2014, p. 30), que implica el desarrollo de acciones encaminadas a la prevención y atención de la discapacidad.

EI País (2020), contabiliza en México 71,130 personas que han estudiado una carrera profesional en el área de la salud, de las cuales el $45.6 \%$ estudiaron en el área de la fisioterapia, terapia física o rehabilitación siendo aproximadamente 32,435 lo que representa que por cada fisioterapeuta o rehabilitador se tiene una demanda de atención de 3932 personas considerando la población total en México de 127,540,408 habitantes. Se han reportado 104 universidades o centros educativos distribuidos en 28 entidades federativas que ofertan una carrera profesional en el área de fisioterapia, rehabilitación o terapia física (EI País, 2020); de estas, cinco entidades no cuentan con una oferta educativa 
dentro de su sistema educativo; y las entidades federativas con mayor presencia de instituciones que ofertan este servicio educativo son Puebla (14\%), Distrito Federal (12\%), México $(8 \%)$ y Yucatán (7\%), lo que representa el $32.49 \%$ del total nacional. En general podría ser que la oferta educativa es insuficiente, por mencionar un ejemplo, el estado de Nuevo León como entidad federativa solo cuenta con dos universidades privadas que ofertan un programa de estudios en el nivel superior para atender a una población de $5,119,504$ habitantes.

La actividad física y deportiva se ve monitoreada por el Módulo de Práctica Deportiva y Ejercicio Físico (INEGI, 2021), los resultados de la encuesta más reciente previa a la pandemia por COVID-19, indicaron que un $42.1 \%$ de la población en México era activa practicando actividad física o deportiva. Adicionalmente, se ha reportado que el $29 \%$ de la población de 20 a 69 años realiza menos de 150 minutos de actividad física por semana y en contraste, el 20\% realiza más de 1680 minutos semanales de actividad física (Secretaría de Salud, 2018). En relación al deporte, un representante de la Comisión Nacional de Cultura Física y Deporte, señala que en el país los deportes más vulnerables para sufrir lesiones son los que se realizan en conjunto por la cantidad de atletas que reúnen y el número de juegos que pueden llegar a tener en una competencia; en segundo lugar, identificó a los deportes de combate; y en tercer lugar, los deportes que conllevan un sobreuso extremo de tejidos, identificados como deportes de resistencia (Comisión Nacional de Cultura Física y Deporte, 2017). Esto implica la posibilidad de padecer algún un tipo de lesión, en algún momento de la práctica lo que abre la posibilidad de requerir algún tratamiento de recuperación o rehabilitación donde intervenga un especialista en el área de la terapia física y/o deportiva.

\section{Oferta laboral}

Partiendo del análisis de 30 planes de estudio de universidades e instituciones educativas, se encontró una oferta educativa con 32 opciones para que sus egresados incursionen y se incorporen laboralmente. Para los análisis se agrupó a aquellas opciones con una representatividad mínima en la categoría de "otros" que agrupa 10 opciones; en suma, se identifican al menos 13 opciones laborales para este tipo de profesionales. 


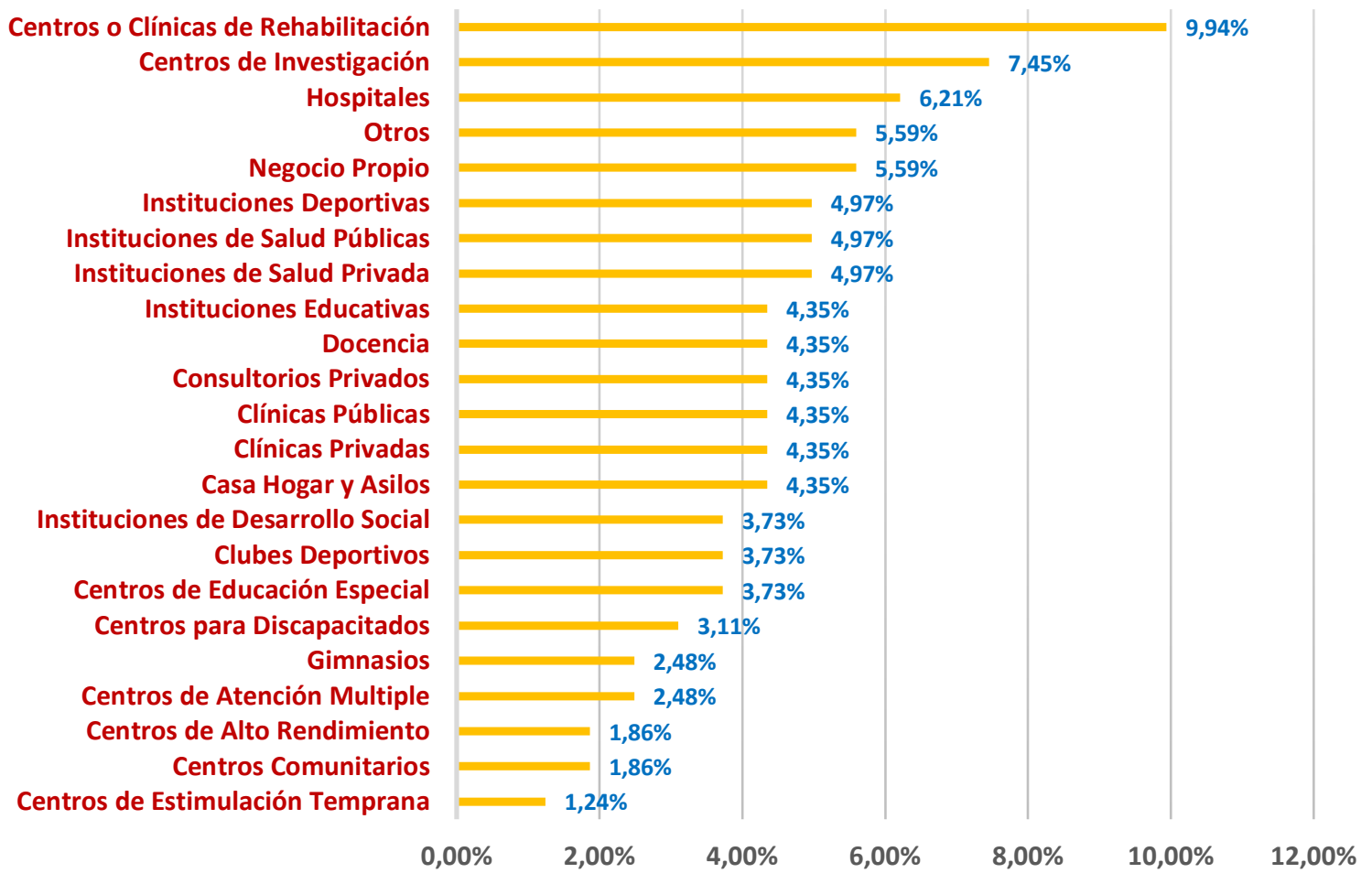

Figura 1. Oferta laboral considerada por las universidades e instituciones educativas que imparten Fisioterapia, Terapia física y rehabilitación. Nota: Los datos proporcionados en esta gráfica fueron generados a partir del análisis descriptivo de contenidos de 33 Universidades e Instituciones con una oferta educativa en fisioterapia, terapia física y rehabilitación (lo que corresponde con un $45.45 \%$ del total de los programas considerados). Fuente: Elaboración propia.

Al respecto del perfil profesional de los fisioterapeutas, Gómez, Silva y David (2015) destacan algunos campos ocupacionales de acción que proponen organizaciones internacionales como la Confederación Mundial de Fisioterapia (WCTP) y la Asociación Americana de Fisioterapia (APTA). De acuerdo con esas organizaciones el campo de acción de los fisioterapeutas incluye la promoción de la salud, la prevención de la enfermedad, la asistencia terapéutica, la habilitación y rehabilitación, la educación, la administración, la gestión, investigación e implementación de políticas de salud a nivel local. Por otro lado, la Dirección General de Profesiones identifica los siguientes títulos profesionales protegidos por la ley en México: Licenciatura en Fisioterapia, Licenciatura en Terapia Física y Licenciatura en Terapia Física y Rehabilitación; esta Dirección señala que los fisioterapeutas pueden: actuar como primer contacto (practicantes autónomos), evaluar pacientes, hacer un diagnóstico, referir a otros especialistas/servicios, tratar y proporcionar servicios de telesalud (World Physiotherapy, 2020). Una limitación que deben enfrentar los 
egresados de esta carrera en el sistema público de México es que todos los pacientes deben ser referidos por el médico especialista o por el médico familiar en el primer nivel de atención mientras que, en la práctica privada, el paciente puede acudir al fisioterapeuta sin ser derivado por un médico. En el Instituto Mexicano del Seguro Social, la institución de salud pública que abarca la atención de la mayoría de la población en el país, las acciones del fisioterapeuta descritas en el manual de procedimientos no coinciden con las marcadas en su perfil de egreso universitario en base a sus competencias (Santamaría Damián, Martínez Olguín, Reséndiz Ramírez, Pacheco Soto y Jaramillo-Díaz, 2012); en el 2021 esta discrepancia sigue afectando el desarrollo profesional en el entorno laboral y de cierta forma, el prestigio social de la importancia de esta profesión.

Según el censo anual que publica la organización World Physiotherapy Mexico (WPM) por cada cinco millones de habitantes se estima que en Sudamérica existen en promedio 14.3 programas educativos relativos a esta rama, sin embargo México solo cuenta con 9.4 programas educativos a este respecto; a nivel global el $63 \%$ de las personas que practican la fisioterapia son mujeres, mientras que en México este porcentaje es superior (70\%); sólo se identifican en este país de uno a cinco fisioterapeutas por cada 10,000 habitantes (WPM, $\underline{2020)}$.

Por su naturaleza, la profesión proporciona una opción de emprender un negocio propio a través de la creación de una clínica de rehabilitación física y fisioterapia en el deporte, aspecto que las universidades e instituciones consideran dentro del campo laboral como la cuarta opción para un estudiante que egresa, según el análisis realizado a los diferentes planes de estudio de 30 entidades educativas que ofertan esta profesión. Uribe (2015) plantea un modelo de negocio enfocado a la rehabilitación y terapia física o deportiva que se fundamenta en la densidad de la población mexicana que práctica actividad física o algún deporte en edades desde los 18 hasta los 80 años como el mercado objetivo según datos proporcionados por el INEGI en el 2021 había una población activa del $42.10 \%$. El modelo de Uribe plantea el emprendimiento de un negocio propio con una clínica de rehabilitación física y el deporte en un horario de 8:00 a.m. a 21:00 p.m. de lunes a vienes y de 8:00 a.m. a 14:00 p.m. el sábado; con una oferta de diferentes modalidades terapéuticas como electroterapia, hidroterapia, termoterapia y mecanoterapia considerando cinco fisioterapeutas en dos turnos; además, sugiere una infraestructura de un local de 200 $\mathrm{m}^{2}$, cerca de oficinas, centros comerciales y gimnasios, con una inversión inicial de US $\$ 60,450$, con un margen de utilidad del $24 \%$ al $34 \%$. A este segmento se suma el de las personas con dificultades para el movimiento, que constituyen el $58.8 \%$ de los 5.7 millones 
de personas con discapacidad en México (INEGI, 2015), mercado en el que al menos dos de cada 10, acude a clínicas particulares.

\section{Pertinencia socioeconómica}

Uno de los aspectos que compete y es de interés en carrera de terapia física, fisioterapia o rehabilitación se centra sobre la oferta educativa y laboral en relación con la remuneración económica que retribuye la profesión con base en el tiempo de inversión en el proceso de formación por una parte y por la otra, la demanda de los servicios laborales que requiere el contexto social. El portal el País $(\underline{2020})$ establece que el $0.71 \%$ de la población en México estudia una carrera en el área de la salud de los cuales el $0.32 \%$ se encuentran en el área disciplinar de la terapia física, la fisioterapia o rehabilitación, con una condición laboral del $98 \%$ en la tasa de ocupación por encima del promedio nacional que es del $95.8 \%$. Desde otro ángulo, esta carrera presenta tan solo un $2 \%$ de tasa de desempleo que según los datos proporcionados por este portal se encuentran por debajo del promedio nacional que es de $4.2 \%$ lo que la hace una profesión con un alto índice de ocupación.

En relación con la remuneración económica, es la novena carrera mejor pagada con un salario mensual promedio de $\$ 436.86$ dólares estadounidenses, con un retorno de la inversión del gasto en educación que se ubica en el $8.35 \%$ para quienes estudiaron en una universidad pública y del $1.59 \%$ para quienes lo hicieron en una universidad privada. Por otra parte, la Secretaría de Salud en su tabulador de sueldos de la rama de la salud que tiene vigencia del 18 de mayo de $\underline{2018}$ establece que un terapista debe de ganar $\$ 836.63$ dólares sueldo bruto mensual, un terapista especializado se ubica en \$886.12 dólares de sueldo bruto mensual, mientras que un terapista profesional en rehabilitación percibe $\$ 1358.98$ dólares. Mientras que en la Ciudad de México un terapeuta físico gana entre $\$ 402.78$ y $\$ 453.13$ dólares estadounidenses (Figura 2). 


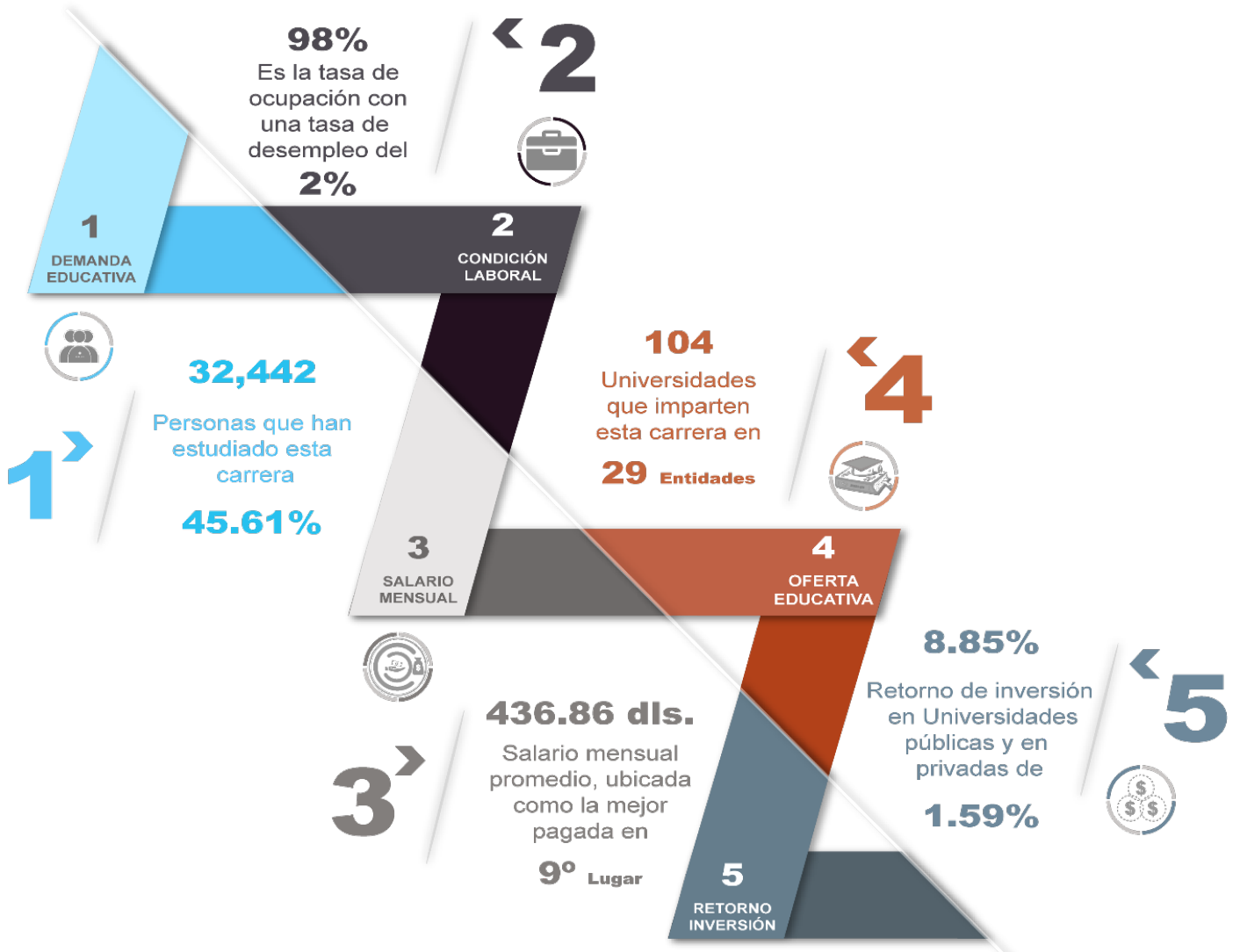

Figura 2. Aspectos socioeconómicos de lo que implica estudiar fisioterapia, terapia física y rehabilitación en México. Nota: La infografía resume cinco aspectos relevantes: la demanda educativa, condición laboral de la carrera, salario mensual promedio, la oferta educativa y el retorno de inversión, que inciden en la toma de decisión para la elección de una carrera profesional en fisioterapia, terapia física y rehabilitación en México. Fuente: Elaboración propia a partir de datos publicados por El País (2020).

\section{CONCLUSIONES}

De acuerdo con este análisis, la oferta educativa nacional de la terapia física se limita a 104 entidades reconocidas que forman profesionales en el ámbito de la terapia y rehabilitación física. En base a la información obtenida y ante la necesidad especifica de la atención de otras poblaciones como las personas con enfermedades crónicas degenerativas no trasmisibles a través de la prescripción del ejercicio o bien a quienes practican deportes de alto rendimiento, la situación es preocupante dado que pudiera implicar la especialización posterior a los estudios fundamentales de terapia física. De considerar que en el 2019 un $42.1 \%$ de la población practicaba algún tipo de actividad física y corre el riesgo de tener alguna lesión, la incursión de la terapia física en el deporte mediante nuevos modelos de readaptación física con rehabilitación y fisioterapia, se 
constituyen como indicadores que otorgan pertinencia a la creación de programas de estudios específicos en el área de la terapia física y deportiva.

En el campo laboral las Universidades consideran al menos 13 opciones donde los egresados pueden desempeñarse profesionalmente, además de contar esta profesión con una rentabilidad sobre el retorno de la inversión de $8.35 \%$ en una universidad pública y de $1.59 \%$ en una universidad privada lo que lo hace muy factible y atractivo en las instituciones educativas públicas. En relación con la demanda laboral en México, esta profesión se posiciona en un $98 \%$ al respecto de la tasa de ocupación, lo cual está por encima del promedio nacional $(95.8 \%)$ y proporciona la oportunidad de emprender un modelo de negocio de forma independiente. En base a lo anterior, la educación universitaria en fisioterapia física y deportiva en México representa un área de oportunidad para las instituciones educativas del país.

\section{REFERENCIAS}

Comisión Nacional de Cultura Física y Deporte. (2017). Lesiones deportivas, un obstáculo a vencer: **Las disciplinas más vulnerables son las de conjunto y contacto // Boletín 2667. Recuperado de https://www.gob.mx/conade/prensa/lesiones-deportivas-unobstaculo-a-vencer

Cortés-González, R. E. y Ravelo-Izquierdo, M. A. (2017). La fisioterapia en el proceso de readaptación físico-deportiva mediante el entrenamiento en un gimnasio con un paciente posoperado de reconstrucción de ligamento cruzado anterior. Caso Clínico. Entre Ciencias: Diálogos en la Sociedad del Conocimiento, 5(12). Recuperado de https://www.redalyc.org/articulo.oa?id=457650040010

Diario Oficial de la Federación [DOF]. (2014). Programa Nacional para el Desarrollo y la Inclusión de las Personas con Discapacidad 2014-2018. Recuperado de http://www.dof.gob.mx/nota detalle.php?codigo=5343100\&fecha=30/04/2014

Encuesta Nacional de Ingresos y Gastos de los Hogares [ENIGH]. (2013). Estadísticas a Propósito del Dia Internacional de las Personas con Discapacidad. Recuperado de https://www.gob.mx/cms/uploads/attachment/file/9640/inegi 2012.pdf

Estrada-Gómez, C. G., Camacho, C., Sánchez, I. y Ochoa, L. (2019). Conocimiento y percepciones médicas sobre la fisioterapia y el trabajo interdisciplinario. Revista Investigación en Educación Médica, 8(31), 38-47. doi: http://dx.doi.org/10.22201/facmed.20075057e.2019.31.18109 
Grindem, H., Granan, L.P., Risberg, M.A., Engebretsen, L., Snyder-Mackler, L. y Eitzen, I. (2015). How does a combined preoperative and postoperative rehabilitation program influence the outcome of ACL reconstruction 2 years after surgery? A comparison between patients in the Delaware-Oslo ACL Cohort and the Norwegian Knee Ligament Registry. British Journal of Sports Medicine, 49(6), 385-389. doi: http://dx.doi.org/10.1136/bjsports-2014-093891

Gómez, E., Silva, V. A., y David, J. (2015). Perfil profesional y ocupacional de los fisioterapeutas en Colombia. CES Movimiento y Salud, 3(1), 35-43.

Instituto Nacional de Estadística y Geografía [INEGI]. (2013). Estadísticas a Propósito del Dia Internacional de las Personas con Discapacidad. Recuperado de https://www.gob.mx/cms/uploads/attachment/file/9640/inegi 2012.pdf Instituto Nacional de Estadística y Geografía [INEGI]. (2015). Censos y Conteos de Población y Vivienda, Encuesta intercensal. Recuperado de https://www.inegi.org.mx/temas/estructura/

Instituto Nacional de Estadística y Geografía [INEGI]. (2021). Módulo de Práctica Deportiva y Ejercicio Físico (MOPRADEP). Recuperado de https://www.inegi.org.mx/programas/mopradef/

Landinez Parra, N. S., Contreras Valencia, K. y Castro Villamil, Á. (2012). Proceso de envejecimiento, ejercicio y fisioterapia. Revista Cubana de Salud Pública, 38(4), 562580. Recuperado de http://scielo.sld.cu/scielo.php?script=sci abstract\&pid=S086434662012000400008

Massimo-Biancardi, C., Luisa-Bona, R. y Lagos-Hausheer, L. (2020). Locomoción humana: modelos y variables biomecánicas. Pensar en Movimiento: Revista de Ciencias del $\begin{array}{lllll}\text { Ejercicio } y & \text { la } & \text { Salud, } & \text { 18(2), }\end{array}$ https://doi.org/10.15517/pensarmov.v18i2.41360

Moreau, W. J. y Nabhan, D. (2012). Organización y trabajo multidisciplinario en un centro olímpico de alto rendimiento en los Estados Unidos. Revista Médica Clínica Las Condes, 23(3), 343-348. doi: https://doi.org/10.1016/S0716-8640(12)70320-6

Organización Mundial de la Salud. (2020). Discapacidad y salud. Recuperado de https://www.who.int/es/news-room/fact-sheets/detail/disability-and-health

Padilla-Muñoz, A. (2010). Discapacidad: contexto, concepto y modelos. International Law: Revista Colombiana de Derecho Internacional, (16), 381-414. Recuperado de https://www.redalyc.org/pdf/824/82420041012.pdf 
El País. (2020, 12 enero). Estudiar en México: terapia y rehabilitación. Recuperado de https://elpais.com/especiales/2015/carreras-mexico/carrera/terapia-yrehabilitacion.html

Pineda, J. A. (2018). El abandono de la rehabilitación física. México: Casa abierta al tiempo. Recuperado

de http://www.evaluacion.azc.uam.mx/assets/abandono rehabilitacion fisica.pdf

Pons, A., Gómez, C., Bueso, P. y Lourido, B. (2006). Relevancia de la formación sobre ejercicio físico y deporte en los planes de estudio de fisioterapia. Fisioterapia, 28(6), 291-297. Recuperado de https://bit.ly/3wA1wMx

Sánchez-Vallejo, P. G., Pérez-Núñez, R. y Heredia-Pi, I. (2015). Costo económico de la discapacidad causada por lesiones de tránsito en México durante 2012. Cadernos de Saúde Pública, 31(4), 755-766. doi: https://doi.org/10.1590/0102-311X00020314

Santamaría Damián, A., Martínez Olguín, M., Reséndiz Ramírez, I. A., Pacheco Soto, C. E., y Jaramillo-Díaz, A. P. (2012). Discrepancia entre el perfil de egreso universitario del fisioterapeuta y sus competencias laborales en instituciones públicas de salud en México. Fisioterapia, 34(1), 39-47. doi: https://doi.org/10.1016/j.ft.2011.09.003

Secretaría de Gobernación. (2017). Ficha temática. Personas con discapacidad. Recuperado de https://www.conapred.org.mx/userfiles/files/Ficha\%20PcD\%281\%29.pdf

Secretaría de Salud. (2018). Encuesta Nacional de Salud y Nutrición 2018. Presentación $\begin{array}{llll}\text { de } & \text { resultados. } & \text { Recuperado }\end{array}$ https://ensanut.insp.mx/encuestas/ensanut2018/doctos/informes/ensanut 2018 pre sentacion resultados.pdf

Thomas, J. R. y Nelson, J. K. (2007). Métodos de investigación en actividad física. Badalona, España: Paidotribo.

Uribe, E. (2015, 14 enero). Abre una clínica del deporte y rehabilitación. Recuperado de https://www.entrepreneur.com/article/267787

World Physiotherapy. (2020). Asociación Mexicana de Fisioterapia. Recuperado de https://world.physio/es/membership/mexico

World Physiotherapy Mexico. (2020). Annual Membership Census. Recuperado de https://world.physio/sites/default/files/2020-12/2020-amc-mexico.pdf 